Irish Math. Soc. Bulletin

Number 72, Winter 2013, 57-70

ISSN 0791-5578

\title{
INTERPLAY BETWEEN SPECTRALLY BOUNDED OPERATORS AND COMPLEX ANALYSIS
}

\author{
MARTIN MATHIEU
}

\begin{abstract}
Methods from Complex Analysis have been playing an important role in the study of spectrally bounded and spectrally isometric operators between Banach algebras in the past few years, not only through the subharmonicity of the spectral radius. In this survey, we intend to illustrate this interplay with Operator Theory by some recent advances in the structural investigation of these operators.
\end{abstract}

\section{INTRODUCTION}

The concept of a spectrally bounded operator was used long before the idea was formalised in [18] in connection with the noncommutative Singer-Wermer conjecture. Le Page [17], Pták [30] and Zemánek [36] exploited inequalities of the form $r(f(x)) \leq r(x)$, where $x$ is an element in a Banach algebra $A$ and $r(x)$ stands for its spectral radius, for certain functions $f$ on $A$ to obtain criteria for commutativity in a general sense. Aupetit proved in [3] that a linear mapping $T$ from a Banach algebra $A$ onto a semisimple Banach algebra $B$ satisfying $r(T x) \leq r(x)$ for all $x \in A$ is automatically continuous, thus providing a new proof for Johnson's uniqueness-of-the-complete-norm-topology theorem. His methods heavily used properties of subharmonic functions while a simplified proof by Ransford, see [31] and [32], rests on alternative techniques from Complex Analysis such as the three-circles theorem. More recently, properties of holomorphic self-maps of the open spectral unit ball in an infinite-dimensional Banach algebra have been exploited to obtain several new results on spectrally bounded and spectrally isometric operators, notably by Costara [11]-[13].

2010 Mathematics Subject Classification. 47A65, 47A10, 47B48, 46L05, 31A05.

Key words and phrases. Spectral isometries, spectrally bounded operators, Jordan isomorphisms, $\mathrm{C}^{*}$-algebras, subharmonic functions.

Received on 2-11-2013. 
The present paper aims to illustrate the usefulness of these methods in this particular branch of Operator Theory by discussing several known results leading to some new evidence towards a conjecture on spectral isometries discussed further below.

\section{BASIC PROPERTIES OF SPECTRALLY BOUNDED OPERATORS}

Throughout $A$ and $B$ will denote unital complex Banach algebras. Let $E$ be a linear subspace of $A$. A linear mapping $T: E \rightarrow B$ is called spectrally bounded provided there is a constant $M \geq 0$ such that $r(T x) \leq M r(x)$ for all $x \in E$. In this case, the smallest such constant is called the spectral operator norm of $T$ and denoted by $\|T\|_{\sigma}$. Let us point out right from the start, although the notions strongly resemble the concepts of a bounded linear operator and its operator norm, our theory here will differ a lot from the classical one since, in the noncommutative setting, the spectral radius is neither subadditive nor submultiplicative and moreover, every quasinilpotent element has spectral radius zero. As a result, the set of spectrally bounded operators on a given space $E$ is not even a vector space.

Spectrally bounded operators were introduced in [18], partly motivated by their connection to the noncommutative Singer-Wermer conjecture and Kaplansky's problem on invertibility-preserving operators. A detailed discussion of the relations with these deep open questions in Banach algebra theory can be found in [22]; see also [9]. Let us first look at a few examples.

Examples 1. (i) Let $T: A \rightarrow B$ be a linear mapping which is unital (that is, $T 1=1$ ) and preserves invertibility. Then $\sigma(T x) \subseteq$ $\sigma(x)$ for every $x \in A$ and hence $T$ is spectrally bounded with $\|T\|_{\sigma}=1$. (Here and in the following, $\sigma(x)$ stands for the spectrum of $x$.)

(ii) Suppose $A$ is a uniform algebra (that is, $A$ is commutative and $\left\|a^{2}\right\|=\|a\|^{2}$ for all $a \in A$ ). Then every bounded linear operator defined on $A$ is spectrally bounded.

(iii) Let $A=M_{n}(\mathbb{C})$ be the algebra of complex $n \times n$ matrices and let $B=\mathbb{C}$. Then the usual trace on $A$ is spectrally bounded with spectral operator norm equal to $n$.

Typical mappings satisfying the assumptions in Example (i) above are Jordan epimorphisms; these are surjective linear mappings such 
that $T\left(x^{2}\right)=(T x)^{2}$ for every $x$. (It does follow that $T$ preserves the Jordan product of any two elements, $x \circ y=\frac{1}{2}(x y+y x)$, so is indeed a homomorphism of the associated Jordan algebras.) However, the other examples illustrate that, without further hypotheses, there are many more possibilities for spectrally bounded operators.

The systematic study of spectrally bounded operators was begun in [24], based on Schick's thesis [34]. Fundamental to all these, and previous, investigations is Vesentini's Theorem from 1968 [35].

Theorem 2 (Vesentini, 1968). Let $f: D \rightarrow A$ be a holomorphic function defined on an open subset $D \subseteq \mathbb{C}$. Then the mapping $\lambda \mapsto r(f(\lambda))$ is subharmonic on $D$.

Subharmonicity here is simply to be understood in the classical sense: the function is upper semi-continuous and satisfies the local submean inequality. For more details we refer to Ransford's book [32] which also contains a nice proof of Vesentini's Theorem.

Another important ingredient is Zemánek's characterization of the Jacobson $\operatorname{radical} \operatorname{rad}(A)$ of $A$ [36]. We recall that $\operatorname{rad}(A)$ is the intersection of all the kernels of irreducible representations of the Banach algebra $A$ as an algebra of bounded linear operators on a Banach space. If $\operatorname{rad}(A)=0$ the algebra $A$ is said to be semisimple, and $A / \operatorname{rad}(A)$ is always semisimple. For further details, please see [2] or [4].

Theorem 3 (Zemánek, 1977). Let $a \in A$ be such that $r(a+x)=r(x)$ for all $x \in A$. Then $a \in \operatorname{rad}(A)$.

It even suffices that the condition in the above theorem is satisfied for all quasi-nilpotent $x \in A$. Proofs of Theorem 3 can be found in [2, Theorem 5.40] and [4, Theorem 5.3.1].

Let us put these results to good use right away.

Proposition 4. Let $T: A \rightarrow B$ be a surjective spectrally bounded operator. Then $\operatorname{Trad}(A) \subseteq \operatorname{rad}(B)$.

The following is just one of the many known arguments; for an alternative one, see [24, Proposition 2.11].

Proof. Suppose that $a \in \operatorname{rad}(A)$; then $r(\lambda a+x)=r(x)$ for all $x \in A, \lambda \in \mathbb{C}$. Fix $y \in B$ and choose $x \in A$ such that $y=$ $T x$. Then $r(\lambda T a+y) \leq\|T\|_{\sigma} r(x)$ all $\lambda \in \mathbb{C}$ so that Liouville's Theorem for subharmonic functions [2, Corollary 5.43] entails that 
$\lambda \mapsto r(\lambda T a+y)$ is constant. Evaluating at $\lambda=1$ and $\lambda=0$ we obtain that $r(T a+y)=r(y)$ for all $y \in B$ and by Theorem 3 , $T a \in \operatorname{rad}(B)$.

Since $r(x+\operatorname{rad}(A))=r(x)$ for every $x \in A$, the above proposition enables us to reduce the study of a surjective spectrally bounded operator $T$ to the semisimple case as

$\hat{T}: \hat{A}=A / \operatorname{rad}(A) \rightarrow \hat{B}=B / \operatorname{rad}(B), \hat{T}(x+\operatorname{rad}(A))=T x+\operatorname{rad}(B)$

is surjective and spectrally bounded with $\|\hat{T}\|_{\sigma}=\|T\|_{\sigma}$.

A far more sophisticated application of subharmonicity enabled Aupetit in [3] to prove the following beautiful result which is by now widely known as Aupetit's Lemma, cf. [2, Theorem 5.45].

Theorem 5 (Aupetit, 1982). For every spectrally bounded operator $T: A \rightarrow B$, the following inequality holds

$$
r(T a) \leq r(T a+y) \text { for all } a \in A \text { and } y \in \mathcal{S}(T),
$$

where $\mathcal{S}(T)=\left\{y \in B \mid y=\lim _{n \rightarrow \infty} T x_{n}, x_{n} \rightarrow 0\right\}$ stands for the separating subspace of $T$.

As a consequence of this, we have the following automatic continuity result.

Corollary 6. For every surjective spectrally bounded operator $T: A \rightarrow B, \mathcal{S}(T) \subseteq \operatorname{rad}(B)$; thus $T$ is bounded if $B$ is semisimple.

Proof. Take $y \in \mathcal{S}(T)$; by Aupetit's Lemma, $r(b) \leq r(b+y)$ for all $b \in B$ as $T$ is surjective. For fixed $b \in B$, pick $x \in A$ with $T x=-(b+y)$ to obtain $r(b+y)=r(T x) \leq r(b)$. Thus $r(y+b)=r(b)$ for all $b$ and Zemánek's characterization of the radical yields the claim. It follows that $\mathcal{S}(T)=0$ in case that $B$ is semisimple; the Closed Graph Theorem now yields that $T$ is bounded.

We will next take a look at a very concrete class of operators, the so-called elementary operators.

For $a \in A$, let $L_{a}$ and $R_{a}$, respectively denote left multiplication $x \mapsto a x$ and right multiplication $x \mapsto x a$, respectively on $A$. As $r(a x)=r(x a)$ for all $x$, spectral boundedness for $L_{a}$ is the same as for $R_{a}$. The following result belongs to Pták [30, Proposition 2.1].

We shall denote by $\mathcal{Z}(A)$ the centre modulo the radical of $A$, that is, the closed subalgebra of $A$ consisting of all $y \in A$ such that $x y-y x \in \operatorname{rad}(A)$ for all $x \in A$. If $A$ is semisimple, this is nothing but the usual centre of $A$. 
Proposition 7. For each $a \in A$, the operator $L_{a}$ is spectrally bounded if and only if $a \in \mathcal{Z}(A)$.

Proof. It is well known that, if $a$ and $x$ commute, then $r(a x) \leq$ $r(a) r(x)$, see, e.g., [4, Corollary 3.2.10]. Since the induced left multiplication $L_{\hat{a}}$ on $\hat{A}=A / \operatorname{rad}(A)$ is spectrally bounded if and only if $L_{a}$ is spectrally bounded, the "if"-part in the proposition follows.

In order to establish the "only if"-part suppose that $r(a x) \leq$ $M r(x)$ for all $x$ and some $M>0$. Fix $x \in A$. Let $\lambda \in \mathbb{C}$ be such that $|\lambda|>M+r(x)$. Write

$$
\lambda-a-x=\left(1-a(\lambda-x)^{-1}\right)(\lambda-x)
$$

and note that

$$
r\left(a(\lambda-x)^{-1}\right) \leq M r\left((\lambda-x)^{-1}\right) \leq M \frac{1}{|\lambda|-r(x)}<1
$$

whence $\lambda-(a+x)$ is invertible.

It follows that $r(a+x) \leq M+r(x)$. In particular, the function $\lambda \mapsto r\left(a-e^{\lambda x} a e^{-\lambda x}\right)$ is bounded on $\mathbb{C}$. Since $f(\lambda)=\frac{1}{\lambda}\left(a-e^{\lambda x} a e^{-\lambda x}\right)$ is entire and $r(f(\lambda))$ is small for large $|\lambda|$, we have $r(f(\lambda))=0$ for all $x \in A$, by Liouville's Theorem. Evaluation at $\lambda=0$ yields

$$
r(a x-x a)=0 \quad(x \in A) .
$$

Suppose there exists an irreducible representation $(\pi, E)$ of $A$ such that $\pi(a) \notin Z(\pi(A))=\mathbb{C} 1$. Then there is $\xi \in E$ such that $\{\xi, \pi(a) \xi\}$ is linearly independent. By Jacobson's Density Theorem [2, Theorem 5.19], there exists $x \in A$ with $\pi(x) \xi=0$ and $\pi(x) \pi(a) \xi=-\xi$. As a result, $(\pi(a) \pi(x)-\pi(x) \pi(a)) \xi=\xi$ which implies that $r(\pi(a) \pi(x)-\pi(x) \pi(a)) \geq 1$, a contradiction. Consequently, $a x-x a \in \operatorname{rad}(A)$ for all $x \in A$ which was to show.

Let $M_{a, b}=L_{a} R_{b}$ denote the two-sided multiplication by $a, b \in A$. An elementary operator on $A$ is simply a linear combination of twosided multiplications, hence of the form

$$
S=\sum_{j=1}^{n} M_{a_{j}, b_{j}}
$$

for a finite subset $\left\{a_{1}, \ldots, a_{n}, b_{1}, \ldots, b_{n}\right\}$ of $A$. By the above result, $M_{a, b}$ is spectrally bounded if and only if $b a \in \mathcal{Z}(A)$. It was shown in [10] that the generalised inner derivation $L_{a}-R_{b}$ is spectrally bounded if and only if both $a \in \mathcal{Z}(A)$ and $b \in \mathcal{Z}(A)$. However, for more general elementary operators, the problem is much harder. 
In [7] we gave a complete characterisation of when $M_{a, b}+M_{c, d}$, an elementary operator of length 2 , is spectrally bounded. A sufficient condition is that both $b a$ and $d c$ belong to $\mathcal{Z}(A)$ and that $d a=0$. For arbitrary length, the problem is still open but in [8] the following sufficient condition was found: $S$ as in (2) is spectrally bounded provided $b_{i} a_{i} \in \mathcal{Z}(A)$ for all $1 \leq i \leq n$ and $b_{j} a_{i}=0$ for all $j>i$. The additional difficulties are largely due to the non-uniqueness of the representation of $S$ by a sum of two-sided multiplications as in (2).

There is a large variety of further general results on spectrally bounded operators for which we refer to the extensive literature (see, e.g., [22] and the references therein). Some reveal a surprisingly strong compatibility with the algebra structure. As a sample, we quote from [25]: If $T$ is a surjective spectrally bounded operator onto a semisimple Banach algebra and $x \in A$ is nilpotent with $x^{n}=0$, then $(T x)^{n}=0$.

We will now turn our attention to a special class of spectrally bounded operators.

\section{SPECTRAL ISOMETRIES}

A linear mapping $T: A \rightarrow B$ is called a spectral isometry if $r(T x)=$ $r(x)$ for every $x \in A$. It can be shown, see [27] e.g., that, under the additional assumption of surjectivity, $\operatorname{Trad}(A)=\operatorname{rad}(B)$ and hence $B$ is semisimple if $A$ is. Moreover, if $A$ is semisimple, $T$ is injective [24, Proposition 4.2] and therefore, $T^{-1}: B \rightarrow A$ is also a bijective spectral isometry. It now follows from Corollary 6 together with Banach's Homomorphism Theorem that $T$ is a topological isomorphism between $A$ and $B$. It is close at hand to wonder how much more of the structure such $T$ may preserve. As it is possible to reduce the general case to the one when $T$ is unital, we will make henceforth this additional assumption which allows us to state the following conjecture first formulated in [24].

Conjecture 8. Every unital surjective spectral isometry between unital $C^{*}$-algebras is a Jordan isomorphism.

It is well possible that this conjecture even holds in the setting of semisimple Banach algebras; if this is the case, it would imply a positive answer to Kaplansky's question on spectrum-preserving linear mappings for which so far only partial answers are known; 
see, e.g., [5]. Indeed, no counter examples are known and there are recent results, see e.g. [1] and [14], providing an affirmative answer under additional hypotheses.

The conjecture is, of course, partly motivated by the classical Banach-Stone Theorem (which covers the commutative situation) and Kadison's generalization to unital isometries between general $C^{*}$-algebras [16].

Theorem 9 (Kadison, 1951). Every unital surjective isometry between two unital $C^{*}$-algebras is a Jordan ${ }^{*}$-isomorphism.

The difference between the classical situation of isometries (with respect to the norm) and our situation is precisely the issue of preservation of the self-adjoint elements: a unital surjective spectral isometry $T$ is an isometry if and only if it sends self-adjoint elements in $A$ onto self-adjoint elements in $B$ (it is a *-mapping). One direction follows from Kadison's theorem, the other from an application of the Russo-Dye Theorem. See also [21]

Another motivation comes from Physics which we will try to explain using the diagram below.

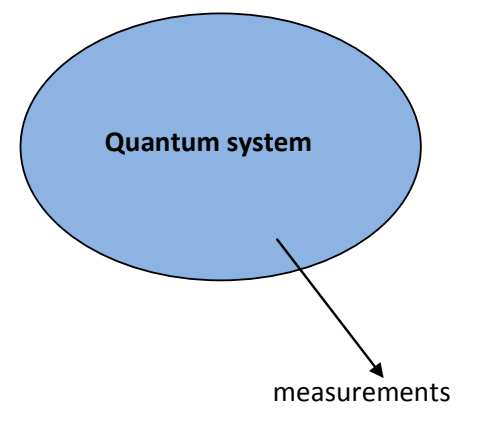

Modelled by C*-algebra A

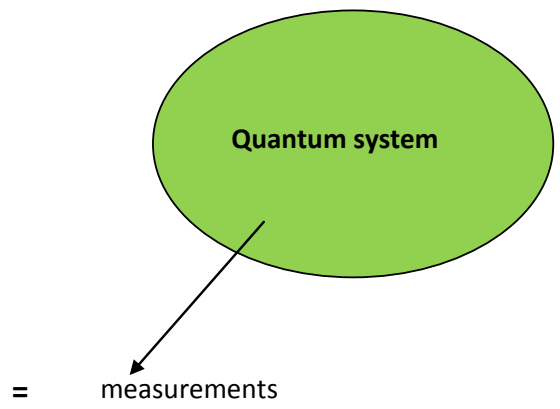

Modelled by C*-algebra B

If $A$ and B are "spectrally isomorphic",

are they isomorphic as models of the same quantum system?

Suppose we model a certain quantum system by a $C^{*}$-algebra $A$ (in the sense that the physical observables of the system are represented by self-adjoint elements in $A$ ). Suppose we do the same with another system and a $C^{*}$-algebra $B$. As we cannot make direct observations in the systems, we entirely rely on the measurements performed on the observables; these relate to the spectral values of the selfadjoint elements. Now suppose all the measurements we take agree 
with one another; in other words, we have a bijective correspondence between the spectra of all (self-adjoint) elements in $A$ and $B$ (which we shall assume to be linear). If the $C^{*}$-algebras $A$ and $B$ are "spectrally isomorphic" in this sense, can we conclude that they are models of the same quantum system? This is a problem of reconstructing the physical system from its data, or from the model that we use to evaluate the data, and leads to Kaplansky's question whether, at least for $C^{*}$-algebras, a bijective spectrum-preserving linear mapping must be a Jordan isomorphism (the Jordan structure being the relevant algebraic structure describing quantum systems). Our conjecture above goes somewhat further: we only require that "the largest eigenvalue" of each element is preserved.

Since Jordan isomorphisms do preserve the spectrum, a test for the above conjecture has to be which parts of the spectrum, or sets related to the spectrum, a unital spectral isometry will preserve. We have the following results [24].

Proposition 10 (Mathieu-Schick, 2002). Let T: $A \rightarrow B$ be a unital surjective spectral isometry, where $A$ is semisimple. Then, for each $a \in A$,

(i) $\sigma_{\text {per }}(T a)=\sigma_{\text {per }}(a)$, where $\sigma_{\mathrm{per}}(a)=\{\lambda \in \sigma(a)|| \lambda \mid=r(a)\}$ is the peripheral spectrum;

(ii) $\operatorname{co} \sigma(T a)=\operatorname{co} \sigma(a)$, where co $\sigma(a)$ is the convex hull of the spectrum.

In particular, singleton spectra are preserved.

Recently, Costara [13] improved this result to the following one.

Proposition 11 (Costara, 2012). Let $T: A \rightarrow B$ be a unital surjective spectral isometry, where $A$ is semisimple. Then, for each $a \in A$,

$$
\sigma(a)=\{\lambda, \mu\} \Longrightarrow \sigma(T a)=\{\lambda, \mu\} .
$$

In order to obtain this result, we have to take a look at some infinite-dimensional Complex Analysis now.

\section{More Complex Analysis}

Let $A$ be a semisimple Banach algebra. By

$$
\Omega_{A}=\{a \in A \mid r(a)<1\}
$$


we denote the open spectral unit ball. Since the spectral radius is upper semi-continuous, $\Omega_{A}$ is open but it need not be convex and will in general not be bounded as there can be quasi-nilpotent elements of arbitrary large norm. Every linear spectral isometry provides us with a holomorphic self-map of $\Omega_{A}$; thus it is close at hand to study mappings of this type when investigating spectral isometries.

Theorem 12 (Ransford, 1996). Let $A$ be a semisimple Banach algebra. Let $F: \Omega_{A} \rightarrow \Omega_{A}$ be holomorphic with $F(0)=0$ and $F^{\prime}(0)=$ id. Then $F(z)=z$ for all $z \in \Omega_{A} \cap Z(A)$.

This Cartan-type theorem differs from the classical one - which states that a holomorphic self-map $F$ on a bounded domain $D$ in $\mathbb{C}$ satisfying $F\left(z_{0}\right)=z_{0}$ and $F^{\prime}\left(z_{0}\right)=1$ for some $z_{0} \in D$ has to be the identity on $D$-in that we do not recover the identity throughout but only on the centre of $A$. In fact, the second part of Ransford's theorem [33] states that for every non-central element $a \in \Omega_{A}$ there is a mapping $F$ satisfying the assumptions of Theorem 12 such that $F(a) \neq a$.

In his paper [13], Costara uses Ransford's theorem to obtain the following very detailed information on the spectral behaviour of selfmaps of the open spectral unit ball which in turn yields a proof of Proposition 11 above.

For $\zeta \in \mathbb{C}$, denote by $D(\zeta, r)$ the open disk centred at $\zeta$ with radius $r>0$ and by $\bar{D}(\zeta, r)$ its closure. Let $\Gamma_{r}=\partial D(\zeta, r)$ be its boundary.

Theorem 13 (Costara, 2012). Let $A$ be a semisimple Banach algebra. Let $F: \Omega_{A} \rightarrow \Omega_{A}$ be holomorphic with $F(0)=0$ and $F^{\prime}(0)=$ id. Suppose that $\bar{D}(\zeta, r) \subseteq \mathbb{D}=D(0,1)$ and that $a \in A$ satisfies $\sigma(a) \subseteq$ $\bar{D}(\zeta, r)$. Then $\sigma(F(a)) \subseteq \bar{D}(\zeta, r)$ and $\sigma(a) \cap \Gamma=\sigma(F(a)) \cap \Gamma$.

Proposition 11 provides us with further evidence towards Conjecture 8: Every Jordan homomorphism sends idempotents to idempotents hence, necessarily, if the spectrum of an element is $\{0,1\}$ then the spectrum of its image under a unital surjective spectral isometry must be $\{0,1\}$ too.

In another paper [12], Costara obtained the following variant of Ransford's theorem above.

Theorem 14 (Costara, 2009). Let $A$ be a primitive Banach algebra. Let $F: \Omega_{A} \rightarrow \Omega_{A}$ be holomorphic with $F(0)=0$ and $F^{\prime}(0)=$ id. Suppose that $F(x) x=x F(x)$ for all $x \in \Omega_{A}$. Then $F=\operatorname{id}_{\Omega_{A}}$. 
Besides Complex Analysis the proof of this result also uses the theory of commuting mappings, and this is essentially the reason why one has to assume that $A$ is primitive. As a consequence of Theorem 14, Costara then establishes the following result, thus partially affirming Conjecture 8 for von Neumann algebras.

Theorem 15 (Costara, 2009). Let $T: A \rightarrow B$ be a unital surjective spectral isometry between von Neumann algebras $A$ and $B$. If Ta and $T\left(a^{2}\right)$ commute for all $a \in A$, then $T$ is a Jordan isomorphism.

To obtain this result, the situation is reduced to the case of primitive algebras by making use of so-called Glimm ideals; these are those closed two-sided ideals which are generated by maximal ideals in the centre of the algebra. In von Neumann algebras, every Glimm ideal is primitive; a well-known result due to Halpern. One can then reduce to primitive quotients by employing the fact that the Glimm ideals separate the points together with the following result taken from [26].

Theorem 16 (Mathieu-Sourour, 2004). Let $T: A \rightarrow B$ be a unital surjective spectral isometry between von Neumann algebras $A$ and $B$. For each Glimm ideal $I \subseteq A, J=T I$ is a Glimm ideal in $B$ and the induced mapping $T_{I J}: A / I \rightarrow B / J$ is a unital surjective spectral isometry.

A key ingredient in the latter result is the spectral radius formula in quotient $C^{*}$-algebras due to Pedersen [29], see also [28]. Another reduction technique using suitable quotients was exploited in our latest paper on the subject, [27], a brief discussion of which shall finish off this survey.

\section{The STRUCTURE OF SPECTRAL ISOMETRIES}

The structure of spectral isometries on commutative algebras is well understood, and it may be close at hand to use this knowledge to unravel it in the noncommutative setting, especially as being a Jordan homomorphism is a local property of a linear mapping.

The following useful observation was obtained in [27]. For an element $a$ in a unital $C^{*}$-algebra $A, C^{*}(a)$ stands for the unital $C^{*}$-subalgebra of $A$ generated by $a$ and $\{a\}^{c c}$ for the bi-commutant (with respect to $A$ ). 
Proposition 17. Let $A$ be a unital $C^{*}$-algebra and let $B$ be a unital Banach algebra. The following conditions on a unital surjective spectral isometry $T: A \rightarrow B$ are equivalent.

(a) $T$ is a Jordan isomorphism;

(b) $T A_{0}$ is a subalgebra of $B$ for every commutative unital subalgebra $A_{0}$ of $A$;

(c) $T\left(\{a\}^{c c}\right)$ is a subalgebra of $B$ for every element $a \in A_{\text {sa }}$;

(d) $T C^{*}(a)$ is a subalgebra of $B$ for every element $a \in A_{s a}$.

If, in the above proposition, $A$ is merely assumed to be a unital semisimple Banach algebra instead of a unital $C^{*}$-algebra, the condition that $T\left(\{a\}^{c c}\right)$ is a subalgebra of $B$ for every element $a \in A$ still yields that $T$ is invertibility preserving.

The main result of [27] is the following.

Theorem 18 (Mathieu-Sourour, 2013). Let $A$ and $B$ be unital semisimple Banach algebras, and let $T: A \rightarrow B$ be a unital surjective spectral isometry. Suppose that $B$ has a separating family $\mathscr{I}$ of closed ideals $I$ such that $B / I$ is semisimple and that each unital surjective spectral isometry from $B / I$ onto $B / I$ is multiplicative or anti-multiplicative. Then $T$ preserves invertibility. If, moreover, $A$ is a $C^{*}$-algebra, then $T$ is a Jordan isomorphism.

The idea of the proof is the following. Let $a \in A$ and put $A_{0}=$ $\{a\}^{c c}$. Let $B_{0}=T A_{0}$. Take $b_{1}, b_{2} \in B_{0}$ and $x \in\{a\}^{c}$, the commutant of $a$. By a series of lemmas we show that $T^{-1}\left(b_{1} b_{2}\right) x+I=$ $x T^{-1}\left(b_{1} b_{2}\right)+I$ for every $I \in \mathscr{I}$. As $\mathscr{I}$ is separating, this yields $T^{-1}\left(b_{1} b_{2}\right) x=x T^{-1}\left(b_{1} b_{2}\right)$ which entails that $T^{-1}\left(b_{1} b_{2}\right) \in A_{0}$, equivalently, $b_{1} b_{2} \in B_{0}$. We conclude that $B_{0}$ is a (closed, unital) subalgebra of $B$. If $A$ is a $C^{*}$-algebra, this argument shows that $T\left(\{a\}^{c c}\right)$ is a subalgebra of $B$ for every element $a \in A_{s a}$. Now Proposition 17 completes the proof. The more general case is covered by the remark following that proposition.

Theorem 18 applies, e.g., to $B=C(X) \otimes M_{n}(\mathbb{C})$ or $B=C(X) \otimes D$, where $D$ is a unital, purely infinite, simple $C^{*}$-algebra (and $X$ is a compact Hausdorff space). It was obtained under the hypothesis that $B$ has a faithful family of finite-dimensional irreducible representation by Costara and Repovš in [14]. 
The next two consequences of Theorem 18 were obtained in [23] under the additional assumption that the spectrum of the domain algebra is totally disconnected.

Corollary 19. Let $T: A \rightarrow B$ be a unital surjective spectral isometry from a unital type I $C^{*}$-algebra with Hausdorff primitive ideal space onto a unital $C^{*}$-algebra $B$. Then $T$ is a Jordan isomorphism.

Corollary 20. Let $A$ and $B$ be unital $C^{*}$-algebras, and let $T: A \rightarrow$ $B$ be a unital surjective spectral isometry. Suppose that $A$ has real rank zero and no tracial states and that its primitive ideal space contains a dense subset of closed points. Then $T$ is a Jordan isomorphism.

Further evidence for the truthfulness of the above conjecture is contained in some of the papers listed below.

\section{REFERENCES}

[1] J. Alaminos, J. Extremera and A. R. Villena, Spectral preservers and approximate spectral preservers on operator algebras, preprint, 2012.

[2] G. R. Allan, Introduction to Banach spaces and algebras, Oxford Graduate Texts in Mathematics 20, Oxford Univ. Press, Oxford, 2011.

[3] B. Aupetit, The uniqueness of the complete norm topology in Banach algebras and Banach Jordan algebras, J. Funct. Anal. 47 (1982), 1-6.

[4] B. Aupetit, A primer on spectral theory, Springer-Verlag, New York, 1991.

[5] B. Aupetit, Spectrum-preserving linear mappings between Banach algebras or Jordan-Banach algebras, J. London Math. Soc. 62 (2000), 917-924.

[6] B. Aupetit And M. Mathieu, The continuity of Lie homomorphisms, Studia Math. 138 (2000), 193-199.

[7] N. Boudi And M. MAthiEu, Elementary operators that are spectrally bounded, Operator Theory: Advances and Applications 212 (2011), 1-15.

[8] N. Boudi ANd M. Mathieu, More elementary operators that are spectrally bounded, preprint, 2013.

[9] M. BrešAr And M. Mathieu, Derivations mapping into the radical, III, J. Funct. Anal. 133 (1995), 21-29.

[10] R. E. Curto And M. Mathieu, Spectrally bounded generalized inner derivations, Proc. Amer. Math. Soc. 123 (1995), 2431-2434.

[11] C. Costara, A Cartan type theorem for finite-dimensional algebras, Linear Alg. Appl. 426 (2007), 299-304.

[12] C. Costara, Commuting holomorphic maps on the spectral unit ball, Bull. London Math. Soc. 41 (2009), 57-62.

[13] C. Costara, On the automorphisms of the spectral unit ball, Proc. Amer. Math. Soc. 140 (2012), 4181-4186.

[14] C. Costara And D. Repovš, Spectral isometries onto algebras having a separating family of finite-dimensional irreducible representations, J. Math. Anal. Appl. 365 (2010), 605-608. 
[15] L. A. HARRIs, Invertibility preserving linear maps of Banach algebras, Contemp. Math. 364 (2004), 59-66.

[16] R. V. KAdison, Isometries of operator algebras, Annals of Math. 54 (1951), 325-338.

[17] C. Le Page, Sur quelques conditions entrainant la commutativité dans les algèbres de Banach, C. R. Acad. Sci. Paris 265 (1967), 235-237.

[18] M. Mathieu, Where to find the image of a derivation, Banach Center Publ. 30 (1994), 237-249.

[19] M. Mathieu, Spectrally bounded operators on simple $C^{*}$-algebras, Proc. Amer. Math. Soc. 132 (2004), 443-446.

[20] M. Mathieu, Spectrally bounded operators on simple $C^{*}$-algebras, II, Irish Math. Soc. Bull. 54 (2004), 33-40.

[21] M. Mathieu, Towards a non-selfadjoint version of Kadison's theorem, in: Proc. Conf. in honour of the 125th birthday of L. Fejér and F. Riesz, Eger, Hungary, June 2005; Ann. Math. Inf. 32 (2005), 87-94.

[22] M. Mathieu, A collection of problems on spectrally bounded operators, Asian-Eur. J. Math. 2 (2009), 487-501.

[23] M. Mathieu and C. Ruddy, Spectral isometries, II, Contemp. Math. 435 (2007), 301-309.

[24] M. Mathieu And G. J. Schick, First results on spectrally bounded operators, Studia Math. 152 (2002), 187-199.

[25] M. Mathieu And G. J. Schick, Spectrally bounded operators from von Neumann algebras, J. Operator Theory 49 (2003), 285-293.

[26] M. Mathieu And A. R. Sourour, Hereditary properties of spectral isometries, Arch. Math. 82 (2004), 222-229.

[27] M. Mathieu And A. R. Sourour, Spectral isometries on non-simple $C^{*}$ algebras, Proc. Amer. Math. Soc. 142 (2014), 129-145.

[28] G. J. Murphy And T. T. West, Spectral radius formulae, Proc. Edinburgh Math. Soc. 22 (1979), 271-275.

[29] G. K. Pedersen, Spectral formulas in quotient $C^{*}$-algebras, Math. Z. 148 (1976), 299-300.

[30] V. PtÁk, Derivations, commutators and the radical, Manuscripta Math. 23 (1978), 355-362.

[31] T. J. RAnsford, A short proof of Johnson's uniqueness-of-norm theorem, Bull. London Math. Soc. 21 (1989), 487-488.

[32] T. J. Ransford, Potential theory in the complex plane, London Math. Soc. Students Texts 28, Cambridge Univ. Press, Cambridge 1995.

[33] T. J. Ransford, A Cartan theorem for Banach algebras, Proc. Amer. Math. Soc. 124 (1996), 243-247.

[34] G. J. Schick, Spectrally bounded operators on Banach algebras, PhD thesis, Queen's University Belfast, 2001.

[35] E. Vesentini, On the subharmonicity of the spectral radius, Boll. Unione Mat. Ital. 4 (1968), 427-429.

[36] J. ZemÁneK, Spectral radius characterizations of commutativity in Banach algebras, Studia Math. 61 (1977), 257-268. 
Dr Martin Mathieu, MRIA has been teaching in several Irish universities such as TCD, UCC (as an Erasmus lecturer) and NUI Maynooth and is presently working at Queen's University Belfast. His research interests include Operator Theory, Operator Algebras and Noncommutative Ring Theory. He is currently the president of the IMS.

Pure Mathematics Research Centre, Queen's University Belfast, Belfast BT7 1NN, Northern Ireland

E-mail address: m.m@qub.ac.uk 